\title{
Perfil do consumo de álcool de usuários de uma unidade de Atenção Primária à Saúde
}

\author{
Profile of users of alcohol consumption of Primary Health Unit
}

Rafael Tavares Jomar', Vitor Augusto de Oliveira Fonseca², Ângela Maria Mendes Abreu ${ }^{3}$, Rosane Harter Griep ${ }^{4}$

\section{Palavras-chave \\ Consumo de bebidas alcoólicas, transtornos relacionados ao uso de álcool, programas de rastreamento, atenção primária à saúde, inquéritos epidemiológicos}

\section{RESUMO}

Objetivo: Descrever o perfil de consumo de álcool de usuários adultos de uma unidade de Atenção Primária à Saúde, segundo características sociodemográficas. Métodos: Inquérito domiciliar desenvolvido com usuários de uma unidade de Atenção Primária à Saúde na cidade do Rio de Janeiro, Brasil. Em 2010, amostra de 301 indivíduos respondeu a um instrumento de coleta que continha o Alcohol Use Disorders Identification Test e variáveis de caracterização sociodemográfica. Análises univariadas com distribuição de frequências simples e bivariadas com diferenças avaliadas pelo teste $X^{2}$ e pelo teste exato de Fisher foram conduzidas, considerando-se um nível de significância de 0,05. Resultados: $O$ consumo de álcool de pessoas do sexo masculino, jovens, de baixa escolaridade, não casadas, empregadas e sem religião mostrou-se mais perigoso para a saúde. Conclusão: Ações preventivas em saúde voltadas para o controle do consumo abusivo de álcool devem estar dirigidas para os grupos mais vulneráveis. É importante enfatizar ações de promoção à saúde de forma a evitar a iniciação e a manutenção de consumo perigoso de álcool, bem como sua evolução para casos de dependência.

\begin{abstract}
Objective: To describe the profile of adult users of alcohol consumption of a unit of Primary Health Care, according to sociodemographic characteristics. Methods: Household survey developed with users of a unit of primary health care in the city of Rio de Janeiro, Brazil. In 2010, sample of 301 individuals responded to a collection instrument containing the Alcohol Use Disorders Identification Test and sociodemographic variables. Univariate analyzes with simple frequency distribution and bivariate with differences evaluated by $X^{2}$ test and Fisher's exact test were conducted considering a 0.05 significance level. Results: The consumption of alcohol males, young and poorly educated, unmarried, employed and no religion was more dangerous to health. Conclusion: Preventive health actions focused on the control of alcohol consumption should be facing these vulnerable groups. It is important to emphasize health promotion actions to prevent the initiation and maintenance of a hazardous alcohol consumption and its evolution for dependency cases.
\end{abstract}

1 Universidade do Estado do Rio de Janeiro (UERJ), Faculdade de Enfermagem, Rio de Janeiro, RJ, Brasil. 2 Universidade Federal do Rio de Janeiro (UFRJ), Faculdade de Odontologia, Rio de Janeiro, RJ, Brasil. 3 UFRJ, Escola de Enfermagem Anna Nery, Rio de Janeiro, RJ, Brasil.

4 Fundação Oswaldo Cruz, Instituto Oswaldo Cruz, Rio de Janeiro, RJ, Brasil.

Endereço para correspondência: Rafael Tavares Jomar Universidade do Estado do Rio de Janeiro, Faculdade de Enfermagem, Departamento de Enfermagem Médico-Cirúrgica Boulevard 28 de Setembro, 157, $6^{\circ}$ andar, Vila Isabel 20551-030 - Rio de Janeiro, RJ, Brasil Telefone: (21) 2868-8236

E-mail: rafaeljomar@yahoo.com.br 


\section{INTRODUÇÃO}

O consumo de álcool é apontado como responsável por grande número de acidentes de trânsito, violência doméstica, aumento da morbimortalidade por doenças cardiovasculares, cirrose hepática e distúrbios psiquiátricos. Seu consumo abusivo está também relacionado a cerca de 2,5 milhões de mortes anuais em todo o mundo, sendo considerado o primeiro fator de risco para a carga global de doenças em países das Américas?.

Mais da metade da população brasileira consome álcool; 25\% dela já experimentaram pelo menos um tipo de problema relacionado ao seu consumo; 3\% preenchem critérios para abuso e 9\%, para dependência ${ }^{2}$. Logo, estudos que identifiquem características sociais, demográficas e individuais associadas ao consumo prejudicial de álcool são necessários para o desenvolvimento de estratégias de redução desse padrão de consumo no Brasi i,4.

No contexto, serviços de Atenção Primária à Saúde (APS) apresentam uma das melhores ocasiões para o rastreamento do uso prejudicial de álcool, além de ser um campo oportuno para o desenvolvimento de ações preventivas voltadas para esse fenômeno ${ }^{5}$. Isso porque a maioria dos pacientes atendidos na APS que consomem álcool em excesso não apresenta, necessariamente, quadro de dependência alcoólica, pois a maior parte dos problemas ocasionados pelo consumo dessa substância manifesta-se entre aqueles que não são dependentes, mas que apresentam comportamento de risco quando bebem ${ }^{6}$.

O conhecimento do estado de saúde de uma comunidade e dos indivíduos que a formam pressupõe entender os seus hábitos e comportamentos de risco, em particular o consumo excessivo de álcool ${ }^{7}$. Por isso, a avalição sistemática do consumo dessa substância, acompanhada de intervenções eficazes, é importante para prevenir consequências sociais e de saúde nas populações, especialmente naquelas atendidas pela Estratégia Saúde da Família (ESF), que tem a promoção da saúde e a prevenção de agravos como práticas sanitárias prioritárias ${ }^{8}$.

A ESF é o modelo assistencial da APS brasileira, fundamentado no trabalho de equipes multiprofissionais em território adstrito, que desenvolve ações de saúde com base no conhecimento da realidade local e das necessidades da população. Tal modelo busca promover o acesso aos serviços, possibilitar o estabelecimento de vínculos entre equipe e usuários e a continuidade do cuidado, além de aumentar a capacidade de resolutividade dos problemas de saúde mais comuns, produzindo maior impacto na situação de saúde local ${ }^{9}$.

As ações preventivas em saúde merecem destaque no atual cenário da saúde pública brasileira. Por isso, a identificação do perfil de consumo de álcool entre usuários de serviços de APS é fundamental, a fim de, pelo menos de modo inicial, oferecer-lhes informações sobre os malefícios de seu uso excessivo, auxiliando na prevenção de agravos causados por ele ${ }^{10}$.

Considerando a escassez e a necessidade de pesquisas sobre o consumo de álcool entre pacientes atendidos no âmbito da APS brasileira, o objetivo deste estudo foi descrever o perfil de consumo de álcool de usuários adultos de uma unidade de APS da cidade do Rio de Janeiro, segundo características sociodemográficas.

\section{MÉTODOS}

Inquérito domiciliar desenvolvido em uma unidade de APS, que funciona no modelo da ESF, situada na zona oeste da cidade do Rio de Janeiro, Brasil. Em 2010, a unidade de saúde em questão era responsável por 587 famílias, que totalizavam 3.476 pessoas, e possuía apenas uma equipe de Saúde da Família (um médico, um enfermeiro, um técnico de enfermagem e seis agentes comunitários de saúde).

Os participantes deste estudo foram selecionados por um processo de amostragem aleatória simples feito no software Epi-Info versão 3.5.1, para cálculo amostral em inquéritos. O tamanho da amostra calculado foi de 279 pessoas, no entanto se planejou entrevistar 307, estimando-se uma perda em torno de $10 \%$ por recusa ou outros motivos.

Para a seleção dos 307 indivíduos, seguiram-se as seguintes etapas: todos os 3.476 prontuários da unidade de APS foram analisados, sendo separados e numerados aqueles pertencentes aos 1.016 adultos cadastrados que atendiam aos critérios de inclusão (idade entre 20-59 anos) e exclusão (indivíduos com incapacidade mental/cognitiva e gestantes) do estudo. Em seguida, foram sorteados os 307 indivíduos, com base nos prontuários numerados previamente.

As visitas aos domicílios não foram agendadas e aconteceram nos meses de novembro e dezembro de 2010: das 9 às 20 horas, de segunda-feira a sexta-feira, e das 9 às 12 horas, aos sábados, domingos e feriados. Os indivíduos foram entrevistados por único pesquisador, em locais reservados e sem a presença de terceiros, por meio de um instrumento de coleta de dados que continha variáveis de caracterização sociodemográfica (sexo, idade, escolaridade, situação conjugal, situação trabalhista e religião) e o Alcohol Use Disorders Identification Test (AUDIT).

Desenvolvido pela Organização Mundial de Saúde ${ }^{11}$ e já validado no Brasil'12, o AUDIT foi escolhido para caracterizar o perfil de consumo de álcool neste estudo, porque ele é o melhor instrumento para ser utilizado em serviços de APS quando comparado a outros com o mesmo fim ${ }^{11,13}$.

O AUDIT é composto por 10 itens: os três primeiros avaliam quantidade, frequência e embriaguez; os três seguintes, sintomas de dependência; e os quatro últimos avaliam o risco de consequências danosas ao usuário. Pelo menos três itens dessa escala são respondidos por todos os indivíduos: 
o primeiro, o nono e o décimo, que questionam sobre a frequência do consumo de álcool, problemas relacionados a ele e sugestões para interrompê-lo, respectivamente. Quando o indivíduo responde o primeiro item afirmando não consumir bebida alcoólica, somente o nono e o décimo item devem ser respondidos e o questionário, então, encerrado.

Respondem ao segundo e terceiro itens do AUDIT aqueles indivíduos que no primeiro afirmam consumir bebida alcoólica. No entanto, caso o somatório dos escores das respostas do segundo e do terceiro item seja igual a 0 (zero), os indivíduos respondem, a partir de então, somente ao nono e décimo itens. Caso contrário (o somatório seja $\geq 1$ ), eles seguem respondendo os itens sequencialmente. Logo, é importante ressaltar que nem todos os indivíduos respondem aos 10 itens do AUDIT.

A seção do instrumento de coleta de dados que se relacionava à caracterização sociodemográfica foi aprimorada por meio de 11 rodadas de pré-testes, quanto ao que se refere à ordem das perguntas e à clareza no entendimento dos entrevistados, em amostra de voluntários que não fizeram parte da amostra final do estudo. Com o objetivo de testar a logística do estudo, foi realizado um estudo piloto com 20 usuários da Unidade Básica de Saúde (UBS) que também não fizeram parte da amostra final deste estudo.

Na fase analítica dos dados, a revisão e a codificação das questões foram realizadas e, em seguida, as informações contidas nos instrumentos de coleta de dados foram digitadas no software Epi-Info versão 3.5.1. No software SPSS versão 13.0 foram realizadas as análises univariadas com distribuição de frequências simples para a descrição da população e também as análises bivariadas.

O perfil de consumo de álcool foi analisado de acordo com as respostas aos itens do AUDIT, segundo as seguintes características sociodemográficas: sexo, faixa etária (20-39 ou 40-59 de idade), escolaridade ( $<8$ anos de estudo ou $\geq 8$ anos de estudo), situação conjugal (casados ou não casados), situação trabalhista (empregados ou não empregados) e religião (possui ou não possui). Considerou-se religioso o indivíduo que respondeu positivamente à questão "Você possui religião atualmente?". As diferenças entre proporções foram avaliadas pelo teste $X^{2} \mathrm{e}$, quando necessário, pelo teste exato de Fisher, considerando-se um nível de significância de 0,05.

O presente estudo foi aprovado pelo Comitê de Ética em Pesquisa da Secretaria Municipal de Saúde e Defesa Civil do Rio de Janeiro, sob o protocolo no 132/2009. Cabe ressaltar que todos os participantes concordaram em participar dele assinando o termo de consentimento livre e esclarecido.

\section{RESULTADOS}

Dos 307 indivíduos selecionados, houve perda de 6 (1,95\%) por recusas na participação do estudo, compondo a amostra final, então, 301 adultos. De modo global, quanto à frequência de consumo de álcool, metade dos entrevistados (50,5\%) afirmou nunca consumi-lo, 10,0\% afirmaram que o consomem de duas a três vezes por semana e 2,3\% afirmaram consumi-lo quatro vezes por semana ou mais.

A análise do perfil de consumo segundo sexo revelou diferenças significativas nos seguintes itens: frequência de consumo $(p=0,002)$, número de doses em dia típico $(p<0,001)$, sentiu-se culpado depois de beber $(p=0,010)$ e alguém sugeriu que parasse de beber ( $p<0,001)$. Em todos eles, as maiores prevalências foram do sexo masculino (Tabela 1).

Tabela 1. Perfil do consumo de álcool de usuários de unidade de APS, segundo sexo (Rio de Janeiro, 2010)

\begin{tabular}{|c|c|c|c|}
\hline Itens do AUDIT & $\begin{array}{c}\text { Homens } \\
n=121(40,2 \%)\end{array}$ & $\begin{array}{c}\text { Mulheres } \\
n=180(59,8 \%)\end{array}$ & p-valor \\
\hline Frequência do consumo & & & 0,002 \\
\hline Nunca & $46(38,0)$ & $106(58,9)$ & \\
\hline Mensalmente ou menos & $21(17,4)$ & $23(12,8)$ & \\
\hline 2 a 4 vezes/mês & $30(24,8)$ & $38(21,1)$ & \\
\hline 2 a 3 vezes/semana & $20(16,5)$ & $10(5,5)$ & \\
\hline 4 ou mais vezes/semana & $04(3,3)$ & $03(1,7)$ & \\
\hline Número de doses em dia típico & & & $<0,001$ \\
\hline 0 & $46(38,0)$ & $106(58,9)$ & \\
\hline $1-4$ & $26(21,5)$ & $48(26,7)$ & \\
\hline 5 ou mais & $49(40,5)$ & $26(14,4)$ & \\
\hline Frequência de seis ou mais doses & & & 0,116 \\
\hline Nunca & $24(32,0)$ & $31(41,9)$ & \\
\hline Mensalmente ou menos & $27(36,0)$ & $30(40,5)$ & \\
\hline Semanalmente ou mais & $24(32,0)$ & $13(17,6)$ & \\
\hline Frequência de beber sem conseguir parar & & & 0,186 \\
\hline Nunca & $40(66,7)$ & $45(77,6)$ & \\
\hline Com alguma frequência & $20(33,3)$ & $13(22,4)$ & \\
\hline Deixou de fazer o esperado devido à bebida & & & 0,215 \\
\hline Nunca & $37(61,7)$ & $42(72,4)$ & \\
\hline Com alguma frequência & $23(38,3)$ & $16(27,6)$ & \\
\hline Necessidade de bebida pela manhã & & & 0,477 \\
\hline Nunca & $50(83,3)$ & $51(87,9)$ & \\
\hline Com alguma frequência & $10(16,7)$ & $07(12,1)$ & \\
\hline Sentiu-se culpado depois de beber & & & 0,010 \\
\hline Nunca & $40(66,7)$ & $25(43,1)$ & \\
\hline Com alguma frequência & $20(33,3)$ & $33(56,9)$ & \\
\hline $\begin{array}{l}\text { Incapaz de lembrar o que aconteceu na noite } \\
\text { anterior por conta da bebida }\end{array}$ & & & 0,536 \\
\hline Nunca & $36(60,0)$ & $38(65,5)$ & \\
\hline Com alguma frequência & $24(40,0)$ & $20(34,5)$ & \\
\hline Feriu-se ou feriu alguém depois de beber & & & 0,646 \\
\hline Nunca & $94(77,7)$ & $143(79,9)$ & \\
\hline Com alguma frequência & $27(22,3)$ & $36(20,1)$ & \\
\hline Alguém sugeriu que parasse de beber & & & $<0,001$ \\
\hline Nunca & $66(54,5)$ & $134(74,4)$ & \\
\hline Com alguma frequência & $55(45,5)$ & $46(25,6)$ & \\
\hline
\end{tabular}


Com relação à idade, a frequência do consumo revelou-se distinta entre as faixas etárias analisadas apenas para o item frequência de seis ou mais doses em única ocasião ( $p=$ $0,014)$, com prevalências mais elevadas entre os mais jovens (Tabela 2).

Tabela 2. Perfil do consumo de álcool de usuários de unidade de APS, segundo faixa etária (Rio de Janeiro, 2010)

\begin{tabular}{|c|c|c|c|}
\hline Itens do AUDIT & $\begin{array}{c}20-39 \text { anos } \\
n=157(52,1 \%)\end{array}$ & $\begin{array}{c}40-59 \text { anos } \\
n=144(47,9 \%)\end{array}$ & p-valor \\
\hline Frequência do consumo & & & 0,350 \\
\hline Nunca & $77(49,0)$ & $75(52,1)$ & \\
\hline Mensalmente ou menos & $20(12,7)$ & $24(16,7)$ & \\
\hline $2 \mathrm{a} 4$ vezes/mês & $40(25,5)$ & $28(19,4)$ & \\
\hline 2 a 3 vezes/semana & $18(11,5)$ & $12(8,3)$ & \\
\hline 4 ou mais vezes/semana & $02(1,3)$ & $05(3,5)$ & \\
\hline Número de doses em dia típico & & & 0,569 \\
\hline 0 & $77(49,1)$ & $75(52,1)$ & \\
\hline $1-4$ & $38(24,2)$ & $36(25,0)$ & \\
\hline 5 ou mais & $42(26,7)$ & $33(22,9)$ & \\
\hline Frequência de seis ou mais doses & & & 0,014 \\
\hline Nunca & $21(26,3)$ & $34(49,3)$ & \\
\hline Mensalmente ou menos & $25(43,7)$ & $22(31,9)$ & \\
\hline Semanalmente ou mais & $24(30,0)$ & $13(18,8)$ & \\
\hline Frequência de beber sem conseguir parar & & & 0,402 \\
\hline Nunca & $51(75,0)$ & $34(68,0)$ & \\
\hline Com alguma frequência & $17(25,0)$ & $16(32,0)$ & \\
\hline Deixou de fazer o esperado devido à bebida & & & 0,163 \\
\hline Nunca & $42(61,8)$ & $37(74,0)$ & \\
\hline Com alguma frequência & $26(38,2)$ & $13(26,0)$ & \\
\hline Necessidade de bebida pela manhã & & & $0,089^{*}$ \\
\hline Nunca & $55(80,9)$ & $46(92,0)$ & \\
\hline Com alguma frequência & $13(19,1)$ & $04(8,0)$ & \\
\hline Sentiu-se culpado depois de beber & & & 0,195 \\
\hline Nunca & $34(50,0)$ & $31(62,0)$ & \\
\hline Com alguma frequência & $34(50,0)$ & $19(38,0)$ & \\
\hline $\begin{array}{l}\text { Incapaz de lembrar o que aconteceu na } \\
\text { noite anterior por conta da bebida }\end{array}$ & & & 0,891 \\
\hline Nunca & $46(63,2)$ & $31(62,0)$ & \\
\hline Com alguma frequência & $25(36,8)$ & $19(38,0)$ & \\
\hline Feriu-se ou feriu alguém depois de beber & & & 0,565 \\
\hline Nunca & $122(77,7)$ & $115(79,9)$ & \\
\hline Com alguma frequência & $35(22,9)$ & $29(20,1)$ & \\
\hline Alguém sugeriu que parasse de beber & & & 0,103 \\
\hline Nunca & $111(70,7)$ & $89(61,8)$ & \\
\hline Com alguma frequência & $46(29,3)$ & $55(38,2)$ & \\
\hline
\end{tabular}

* Teste exato de Fisher.

A análise do perfil de consumo segundo o nível de escolaridade revela, ainda que sem significância estatística, a maior prevalência de indivíduos que consomem álcool quatro ou mais vezes por semana no segmento de menor escolaridade $(3,7 \%$ versus $0,7 \%)$, que também apresenta o percentual mais elevado de sugestões para interromper 0 consumo dessa substância $(p=0,018)$ (Tabela 3).

Tabela 3. Perfil do consumo de álcool de usuários de unidade de APS, segundo escolaridade (Rio de Janeiro, 2010)

\begin{tabular}{|c|c|c|c|}
\hline Itens do AUDIT & $\begin{array}{c}<8 \text { anos de } \\
\text { estudo } \\
n=162 \\
(53,8 \%)\end{array}$ & $\begin{array}{c}\geq 8 \text { anos de } \\
\text { estudo } \\
n=139 \\
(46,2 \%)\end{array}$ & p-valor \\
\hline Frequência do consumo & & & 0,513 \\
\hline Nunca & $82(50,6)$ & $70(50,3)$ & \\
\hline Mensalmente ou menos & $24(14,8)$ & $20(14,4)$ & \\
\hline 2 a 4 vezes/mês & $34(21,0)$ & $34(24,5)$ & \\
\hline 2 a 3 vezes/semana & $16(9,9)$ & $14(10,1)$ & \\
\hline 4 ou mais vezes/semana & $06(3,7)$ & $01(0,7)$ & \\
\hline Número de doses em dia típico & & & 0,569 \\
\hline 0 & $82(50,6)$ & $70(50,4)$ & \\
\hline $1-4$ & $38(23,5)$ & $36(25,9)$ & \\
\hline 5 ou mais & $42(25,9)$ & $33(23,7)$ & \\
\hline Frequência de seis ou mais doses & & & 0,911 \\
\hline Nunca & $29(36,3)$ & $26(37,7)$ & \\
\hline Mensalmente ou menos & $30(37,5)$ & $27(39,1)$ & \\
\hline Semanalmente ou mais & $21(26,2)$ & $16(23,2)$ & \\
\hline Frequência de beber sem conseguir parar & & & 0,072 \\
\hline Nunca & $41(65,1)$ & $44(80,0)$ & \\
\hline Com alguma frequência & $22(34,9)$ & $11(20,0)$ & \\
\hline Deixou de fazer o esperado devido à bebida & & & 0,393 \\
\hline Nunca & $40(63,5)$ & $39(70,9)$ & \\
\hline Com alguma frequência & $23(36,5)$ & $16(29,1)$ & \\
\hline Necessidade de bebida pela manhã & & & 0,124 \\
\hline Nunca & $51(81,0)$ & $50(90,9)$ & \\
\hline Com alguma frequência & $12(19,0)$ & $05(9,1)$ & \\
\hline Sentiu-se culpado depois de beber & & & 0,794 \\
\hline Nunca & $34(54,0)$ & $31(56,4)$ & \\
\hline Com alguma frequência & $29(46,0)$ & $24(43,6)$ & \\
\hline $\begin{array}{l}\text { Incapaz de lembrar o que aconteceu na } \\
\text { noite anterior por conta da bebida }\end{array}$ & & & 0,851 \\
\hline Nunca & $40(63,5)$ & $34(61,8)$ & \\
\hline Com alguma frequência & $23(65,5)$ & $21(38,2)$ & \\
\hline Feriu-se ou feriu alguém depois de beber & & & 0,607 \\
\hline Nunca & $129(79,6)$ & $108(77,7)$ & \\
\hline Com alguma frequência & $33(20,4)$ & $31(22,3)$ & \\
\hline Alguém sugeriu que parasse de beber & & & 0,018 \\
\hline Nunca & $98(60,5)$ & $102(73,4)$ & \\
\hline Com alguma frequência & $34(39,5)$ & $37(26,6)$ & \\
\hline
\end{tabular}

No que tange à situação conjugal, a análise revelou diferenças estatisticamente significativas nos seguintes itens: frequência de consumo $(p<0,001)$, frequência de beber sem conseguir parar ( $p=0,013$ ), incapaz de lembrar o que aconteceu na noite anterior por conta da bebida $(p=0,029)$, feriu-se ou feriu alguém depois de beber $(p<0,001)$ e alguém sugeriu que parasse de beber $(p=0,004)$. Em todos esses 
itens, as maiores prevalências foram entre os indivíduos não casados (Tabela 4).

Tabela 4. Perfil do consumo de álcool de usuários de unidade de APS, segundo situação conjugal (Rio de Janeiro, 2010)

\begin{tabular}{|c|c|c|c|}
\hline Itens do AUDIT & $\begin{array}{l}\text { Casados } \\
\mathrm{n}=197 \\
(65,4 \%)\end{array}$ & $\begin{array}{c}\text { Não casados } \\
n=104 \\
(34,6 \%)\end{array}$ & p-valor \\
\hline Frequência do consumo & & & $<0,001$ \\
\hline Nunca & $111(56,3)$ & $41(39,4)$ & \\
\hline Mensalmente ou menos & $35(17,8)$ & $09(8,7)$ & \\
\hline 2 a 4 vezes/mês & $37(18,8)$ & $31(29,8)$ & \\
\hline 2 a 3 vezes/semana & $12(6,1)$ & $18(17,3)$ & \\
\hline 4 ou mais vezes/semana & $02(1,0)$ & $05(4,8)$ & \\
\hline Número de doses em dia típico & & & 0,669 \\
\hline 0 & $111(56,3)$ & $41(39,4)$ & \\
\hline $1-4$ & $44(22,4 \%)$ & $30(28,9)$ & \\
\hline 5 ou mais & $42(21,3)$ & $33(31,7)$ & \\
\hline Frequência de seis ou mais doses & & & 0,092 \\
\hline Nunca & $38(44,2)$ & $17(27,0)$ & \\
\hline Mensalmente ou menos & $30(34,9)$ & $27(42,8)$ & \\
\hline Semanalmente ou mais & $18(20,9)$ & $19(30,2)$ & \\
\hline Frequência de beber sem conseguir parar & & & 0,013 \\
\hline Nunca & $50(81,9)$ & $35(61,4)$ & \\
\hline Com alguma frequência & $11(18,1)$ & $22(38,6)$ & \\
\hline Deixou de fazer 0 esperado devido à bebida & & & 0,649 \\
\hline Nunca & $42(68,8)$ & $37(64,9)$ & \\
\hline Com alguma frequência & $19(31,2)$ & $20(35,1)$ & \\
\hline Necessidade de bebida pela manhã & & & 0,348 \\
\hline Nunca & $54(88,5)$ & $47(82,5)$ & \\
\hline Com alguma frequência & $07(11,5)$ & $10(17,5)$ & \\
\hline Sentiu-se culpado depois de beber & & & 0,208 \\
\hline Nunca & $37(60,6)$ & $28(49,2)$ & \\
\hline Com alguma frequência & $24(39,4)$ & $29(50,8)$ & \\
\hline $\begin{array}{l}\text { Incapaz de lembrar o que aconteceu na } \\
\text { noite anterior por conta da bebida }\end{array}$ & & & 0,029 \\
\hline Nunca & $44(72,1)$ & $30(52,6)$ & \\
\hline Com alguma frequência & $17(27,9)$ & $27(47,4)$ & \\
\hline Feriu-se ou feriu alguém depois de beber & & & $<0,001$ \\
\hline Nunca & $169(85,8)$ & $69(66,4)$ & \\
\hline Com alguma frequência & $28(14,2)$ & $35(33,6)$ & \\
\hline Alguém sugeriu que parasse de beber & & & 0,004 \\
\hline Nunca & $142(72,1)$ & $58(55,7)$ & \\
\hline Com alguma frequência & $55(27,9)$ & $46(44,3)$ & \\
\hline
\end{tabular}

Com relação à situação trabalhista, os itens frequência de consumo ( $p=0,033)$, número de doses em dia típico ( $p=0,002)$ e frequência de seis ou mais doses $(p=0,051)$ revelaram-se diferentes entre as categorias analisadas, com prevalências mais elevadas entre os indivíduos empregados (Tabela 5).

No que diz respeito à religião, a análise revelou diferenças estatisticamente significativas nos seguintes itens: frequên- cia de consumo ( $p<0,001)$, número de doses em dia típico ( $p=0,014)$, frequência de seis ou mais doses $(p=0,002)$, frequência de beber sem conseguir parar ( $p=0,009)$, feriu-se ou feriu alguém depois de beber $(p<0,002)$ e alguém sugeriu que parasse de beber $(p<0,001)$. Em todos esses itens, as maiores prevalências foram entre aqueles indivíduos que afirmaram não possuir religião (Tabela 6).

Tabela 5. Perfil do consumo de álcool de usuários de unidade de APS, segundo situação trabalhista (Rio de Janeiro, 2010)

\begin{tabular}{|c|c|c|c|}
\hline Variável & $\begin{array}{c}\text { Empregados } \\
n=198 \\
(65,8 \%)\end{array}$ & $\begin{array}{c}\text { Não } \\
\text { empregados } \\
n=103 \\
(34,2 \%)\end{array}$ & $\mathrm{p}$-valor \\
\hline Frequência do consumo & & & 0,033 \\
\hline Nunca & $92(46,5)$ & $60(58,3)$ & \\
\hline Mensalmente ou menos & $26(13,1)$ & $18(17,5)$ & \\
\hline 2 a 4 vezes/mês & $48(24,3)$ & $20(19,4)$ & \\
\hline 2 a 3 vezes/semana & $26(13,1)$ & $04(3,9)$ & \\
\hline 4 ou mais vezes/semana & $06(3,0)$ & $01(0,1)$ & \\
\hline Número de doses em dia típico & & & 0,002 \\
\hline 0 & $92(46,5)$ & $60(58,3)$ & \\
\hline $1-4$ & $44(22,2)$ & $30(29,1)$ & \\
\hline 5 ou mais & $62(31,3)$ & $13(12,6)$ & \\
\hline Frequência de seis ou mais doses & & & $0,051^{*}$ \\
\hline Nunca & $35(33,0)$ & $20(46,5)$ & \\
\hline Mensalmente ou menos & $39(36,8)$ & $18(41,9)$ & \\
\hline Semanalmente ou mais & $32(30,2)$ & $05(11,6)$ & \\
\hline Frequência de beber sem conseguir parar & & & 0,344 \\
\hline Nunca & $64(74,4)$ & $21(65,6)$ & \\
\hline Com alguma frequência & $22(25,6)$ & $11(34,4)$ & \\
\hline Deixou de fazer o esperado devido à bebida & & & 0,115 \\
\hline Nunca & $54(62,8)$ & $25(78,1)$ & \\
\hline Com alguma frequência & $32(37,2)$ & $07(21,9)$ & \\
\hline Necessidade de bebida pela manhã & & & 0,402 \\
\hline Nunca & $75(87,2)$ & $26(81,2)$ & \\
\hline Com alguma frequência & $11(12,8)$ & $06(18,8)$ & \\
\hline Sentiu-se culpado depois de beber & & & 0,568 \\
\hline Nunca & $46(53,5)$ & $19(59,4)$ & \\
\hline Com alguma frequência & $40(46,4)$ & $13(40,6)$ & \\
\hline $\begin{array}{l}\text { Incapaz de lembrar o que aconteceu na } \\
\text { noite anterior por conta da bebida }\end{array}$ & & & 0,189 \\
\hline Nunca & $57(66,6)$ & $17(53,1)$ & \\
\hline Com alguma frequência & $29(33,7)$ & $15(46,0)$ & \\
\hline Feriu-se ou feriu alguém depois de beber & & & 0,862 \\
\hline Nunca & $157(79,3)$ & $81(78,6)$ & \\
\hline Com alguma frequência & $41(20,7)$ & $22(21,4)$ & \\
\hline Alguém sugeriu que parasse de beber & & & 0,241 \\
\hline Nunca & $127(64,1)$ & $73(70,9)$ & \\
\hline Com alguma frequência & $71(35,9)$ & $30(29,1)$ & \\
\hline
\end{tabular}


Tabela 6. Perfil do consumo de álcool de usuários de unidade de APS, segundo religião (Rio de Janeiro, 2010)

\begin{tabular}{|c|c|c|c|}
\hline Variável & $\begin{array}{l}\text { Possui } \\
n=192\end{array}$ & $\begin{array}{c}\text { Não possui } \\
n=109\end{array}$ & p-valor \\
\hline Frequência do consumo & & & $<0,001$ \\
\hline Nunca & $120(62,5)$ & $32(29,3 \%)$ & \\
\hline Mensalmente ou menos & $21(10,9)$ & $23(21,1 \%)$ & \\
\hline 2 a 4 vezes/mês & $45(23,5)$ & $24(22,1 \%)$ & \\
\hline 2 a 3 vezes/semana & $05(2,6)$ & $25(22,9 \%)$ & \\
\hline 4 ou mais vezes/semana & $01(0,5)$ & $05(4,6 \%)$ & \\
\hline Número de doses em dia típico & & & 0,014 \\
\hline 0 & $120(62,5)$ & $32(29,3)$ & \\
\hline $1-4$ & $43(22,4)$ & $30(27,5)$ & \\
\hline 5 ou mais & $29(15,1)$ & $47(43,2)$ & \\
\hline Frequência de seis ou mais doses & & & 0,002 \\
\hline Nunca & $34(47,2)$ & $21(27,3)$ & \\
\hline Mensalmente ou menos & $29(40,3)$ & $27(35,1)$ & \\
\hline Semanalmente ou mais & $09(12,5)$ & $29(37,6)$ & \\
\hline Frequência de beber sem conseguir parar & & & 0,009 \\
\hline Nunca & $44(84,6)$ & $41(62,1)$ & \\
\hline Com alguma frequência & $08(15,4)$ & $25(37,9)$ & \\
\hline Deixou de fazer o esperado devido à bebida & & & 0,965 \\
\hline Nunca & $35(67,3)$ & $44(66,7)$ & \\
\hline Com alguma frequência & $17(32,7)$ & $22(33,3)$ & \\
\hline Necessidade de bebida pela manhã & & & 0,952 \\
\hline Nunca & $45(86,5)$ & $57(86,4)$ & \\
\hline Com alguma frequência & $07(13,5)$ & $09(13,6)$ & \\
\hline Sentiu-se culpado depois de beber & & & 0,739 \\
\hline Nunca & $28(53,8)$ & $38(57,6)$ & \\
\hline Com alguma frequência & $24(46,2)$ & $28(42,4)$ & \\
\hline $\begin{array}{l}\text { Incapaz de lembrar o que aconteceu na } \\
\text { noite anterior por conta da bebida }\end{array}$ & & & 0,668 \\
\hline Nunca & $34(65,4)$ & $41(62,1)$ & \\
\hline Com alguma frequência & $18(34,6)$ & $25(37,9)$ & \\
\hline Feriu-se ou feriu alguém depois de beber & & & 0,002 \\
\hline Nunca & $163(84,9)$ & $75(68,8)$ & \\
\hline Com alguma frequência & $29(15,1)$ & $34(31,2)$ & \\
\hline Alguém sugeriu que parasse de beber & & & $<0,001$ \\
\hline Nunca & $147(76,6)$ & $53(48,6)$ & \\
\hline Com alguma frequência & $45(23,4)$ & $56(51,4)$ & \\
\hline
\end{tabular}

\section{DISCUSSÃO}

O presente estudo descreveu o perfil de consumo de álcool de usuários adultos de uma unidade de APS brasileira, apontando para algumas características sociodemográficas relacionadas ao consumo prejudicial para a saúde. Cabe destacar que a proporção de abstêmios identificados foi semeIhante à de outros estudos brasileiros conduzidos na APS 10,14.

A participação de mulheres foi um pouco maior que a dos homens, que, por sua vez, apresentaram maiores prevalências de consumo prejudicial de álcool, segundo quatro itens do AUDIT. Como fator de risco para o consumo dessa substância entre homens, além do reforço de tal consumo pelos grupos, destaca-se o aspecto do beber como privilégio masculino e uma de suas formas de diversão, bem como uma maneira de estabelecer ou manter interações sociais com o outros de seu entorno, devendo-se ainda ressaltar a liberdade que o meio thes outorga pelo fato de serem homens ${ }^{15,16}$.

No que concerne ao perfil de consumo de álcool segundo as faixas etárias estudadas, a frequência do consumo revelou-se distinta apenas para o item do AUDIT "frequência de seis ou mais doses em única ocasião", com prevalências mais elevadas entre os mais jovens. Indivíduos jovens tendem a consumir álcool em níveis mais elevados ou de maior risco ${ }^{17,18}$. Esse fato pode ser particular da idade, uma vez que as aquisições de responsabilidades pessoais e profissionais, com o avançar da idade, vão se acumulando e tornando o padrão de consumo problemático de álcool incompatível com as atividades rotineiras ${ }^{19}$. Esse grupo é considerado aquele sob os maiores riscos de agravos relacionados ao álcool, de forma que as prevalências dos padrões de consumo nesse segmento demográfico precisam ser especialmente monitoradas ${ }^{20,21}$.

O perfil de consumo de álcool segundo o nível de escolaridade apontou para a maior prevalência de indivíduos que consomem álcool quatro ou mais vezes por semana no segmento de menor escolaridade (3,7\% versus $0,7 \%)$, que também apresenta o percentual mais elevado de sugestões para interromper o consumo dessa substância. A literatura tem demonstrado tendência de maiores taxas de dependência alcoólica em grupos sociais com baixa escolaridade ${ }^{22,23}$ e que ela também pode ser fator importante no envolvimento de pessoas com o uso abusivo ${ }^{16}$.

No que tange à situação conjugal, a análise revelou diferenças estatisticamente significativas em metade dos itens do AUDIT, com maiores prevalências entre os indivíduos não casados. Estudos apontam que indivíduos solteiros apresentam maior chance de manifestar e manter o uso abusivo de álcool24 e que estar casado ou ter companheiro seja fator protetor para o consumo problemático de álcool16,25.

Os itens do AUDIT "frequência de consumo", "número de doses em dia típico" e "frequência de seis ou mais doses" revelaram-se diferentes entre os indivíduos não empregados e aqueles empregados, com prevalências mais elevadas entre os últimos. Outros estudos encontraram associação entre estar empregado e consumo de abusivo de álcool 14,26,27. Acredita-se que o fato de o indivíduo estar empregado e ter salário, portanto, facilite a aquisição de álcool e o consumo de maiores doses. No entanto, vale destacar que essa relação tem sido pouco estudada e necessita de maior investigação no que diz respeito ao maior consumo dessa substância entre a população economicamente ativa ${ }^{27}$.

Em relação à religião, as análises revelaram diferenças estatisticamente significativas em pouco mais da metade dos 
itens do AUDIT, em que as maiores prevalências foram entre aqueles indivíduos que afirmaram não possuir religião. Resultados semelhantes foram encontrados em estudos conduzidos em serviços brasileiros de APS ${ }^{14,28}$, provavelmente, porque muitas religiões, especialmente as de origem cristã, pregam a abstinência alcoólica.

No Brasil, a política nacional de promoção da saúde imprime forte ênfase nas ações educativas e na veiculação de informações sobre os danos do uso abusivo do álcool e propõe iniciativas para a restrição de acesso a bebidas alcoólicas pelos segmentos vulneráveis ${ }^{29}$. Como a proposta da ESF busca ressaltar uma nova concepção de assistência à saúde, por meio de ações preventivas, intervenções sobre os fatores de risco e do desenvolvimento da qualidade de vida ${ }^{9}$, as ações preventivas em saúde, especialmente as de controle do consumo abusivo de álcool, devem estar voltadas para grupos mais vulneráveis: homens, jovens e indivíduos de baixa escolaridade.

Vale destacar que os profissionais de APS ocupam posição relevante na identificação e abordagem daqueles pacientes cujo padrão de consumo de álcool traz risco ou danos para sua saúde 5 . Isso porque o ambiente da APS é ideal para a continuidade do monitoramento e repetição de avaliações e intervenções ${ }^{11}$. Para tanto, é necessário que as instituições formadoras dos profissionais de saúde abordem em suas grades curriculares o consumo prejudicial de álcool e suas consequências, e não somente a dependência alcoólica como único problema decorrente do uso dessa substância. Ademais, a oferta de capacitação aos profissionais atuantes na APS para abordagem dos usuários de álcool com ênfase nas ações de promoção de saúde para grupos de risco também é fundamental.

Diante disso, a utilização de um instrumento fácil de aplicar, válido para obter o padrão do consumo de álcool e diagnosticar sintomas de alcoolismo, constitui importante ferramenta na promoção de maior envolvimento das equipes de saúde, consequente mobilização e proposição de intervenções adequadas ${ }^{4}$. Por isso, a utilização rotineira do AUDIT em serviços de APS é recomendada, por constituir-se em ferramenta útil na identificação do padrão de consumo de álcool, que auxilia, especialmente, médicos e enfermeiros em atividades de proteção e promoção da saúde voltadas aos usuários desses serviços, facilitando sua abordagem em relação ao consumo de bebidas alcoólicas ${ }^{30}$.

Para prevenção primária e secundária do uso prejudicial de álcool, articuladas à aplicação do AUDIT, podem ser realizadas intervenções breves, que são estratégias baseadas em abordagem motivacional com foco na mudança de comportamento do paciente por meio de atendimento curto realizado por profissionais de diferentes formações ${ }^{5,11}$. A intervenção breve é de baixo custo e tem se mostrado efetiva para problemas relacionados ao consumo de álcool, além de constituir-se um meio adequado para referenciar casos corretos de dependência para tratamentos especializados ${ }^{5}$.
Convém mencionar que, apesar de o presente estudo apresentar limitações, tais como a possibilidade da ocorrência do viés de memória, como o desenho de estudo foi apropriado ao que se propunha e o percentual de perdas não foi expressivo (1,95\%), considera-se que a amostra pesquisada foi adequada para a avaliação do perfil de consumo de álcool, segundo características sociodemográficas.

\section{CONCLUSÃO}

Em conclusão, o presente estudo observou que usuários do sexo masculino, jovens, de baixa escolaridade, não casados, empregados e sem religião apresentam consumo de álcool mais prejudicial para a saúde. Tais resultados servem de subsídios para o desenvolvimento e planejamento de ações preventivas ao consumo abusivo de álcool no âmbito da unidade de APS em que foi desenvolvido este estudo. Sugere-se o desenvolvimento de outros estudos sobre o consumo de álcool por usuários de serviços brasileiros de APS com a finalidade de direcionar ações preventivas em saúde específicas para o uso dessa substância, além de colaborar com o aperfeiçoamento de políticas públicas sobre álcool no país.

\section{CONTRIBUIÇÕES INDIVIDUAIS}

Rafael Tavares Jomar - Contribuiu na concepção do projeto e desenho do estudo, coleta e análise de dados, revisão bibliográfica, interpretação dos resultados, redação do artigo, elaboração das tabelas e redação da versão final.

Vitor Augusto de Oliveira Fonseca - Contribuiu na redação do artigo, elaboração das tabelas e redação da versão final.

Ângela Maria Mendes Abreu - Participou como orientadora na concepção do projeto e desenho do estudo, revisão do artigo e de sua versão final.

Rosane Harter Griep - Participou como orientadora na concepção do projeto e desenho do estudo, revisão do artigo e de sua versão final.

\section{CONFLITOS DE INTERESSE}

Os autores não possuem conflitos de interesse a serem declarados.

\section{REFERÊNCIAS}

1. World Health Organization. Global status report on alcohol and health. Geneva: World Health Organization; 2011.

2. Laranjeira R, Pinsky I, Sanches M, Zaleski M, Caetano R. Alcohol use patterns among Brazilian adults. Rev Bras Psiquiatr. 2010;32(3):231-41. 
3. Silveira CM, Silveira CC, Silva JG, Silveira LM, Andrade AG, Andrade LHSG. Epidemiologia do beber pesado e beber pesado episódico no Brasil: uma revisão sistemática da literatura. Rev Psiq Clín. 2008;35(Supl 1):S31-8.

4. Barros MBA, Marin-León L, Oliveira HB, Dalgalarrondo P, Botega NB. Perfil do consumo de bebidas alcoólicas: diferenças sociais e demográficas no município de Campinas, estado de São Paulo, Brasil, 2003. Epidemiol Serv Saúde. 2008;17(4):259-70.

5. Marques ACPR, Furtado EF. Intervenções breves para problemas relacionados ao álcool. Rev Bras Psiquiatr. 2004;26(Supl 1):S28-32.

6. Cordeiro Q, Michelon L, Ribeiro RB, Kamitsuji C, Silveira CM, Andrade LHG. Triagem para a identificação de uso nocivo de álcool na atenção primária à saúde. Rev Assoc Med Bras. 2006;52(4):200.

7. Breda J, de Almeida MDV. Validação de um instrumento de avaliação da ingestão de bebidas alcoólicas e de etanol por consumidores excessivos. Rev Acta Med Port. 2010;23:95564.

8. Abreu AMM, Jomar RT, Souza MHN, Guimarães RM. Consumo prejudicial de álcool entre usuários da Estratégia Saúde da Família. J Bras Psiquiatr. 2013;62(1):91-2.

9. Brasil. Ministério da Saúde. Portaria n².488, de 21 de outubro de 2011. Disponível em: <http://www.prefeitura.sp.gov.br/cidade/secretarias/upload/saude/arquivos/esf/Portaria2488_2011.pdf>. Acesso em: 28 out. 2014.

10. Abreu AMM, Jomar RT, Souza MHN, Guimarães RM. Consumo nocivo de bebidas alcoólicas entre usuários de uma Unidade de Saúde da Família. Acta Paul Enferm. 2012;25(2):291-5.

11. Babor TF, Higgins-Biddle JC, Saunders JB, Monteiro MG. The alcohol use disorders identification test: guideline for use in primary care. 2nd ed. Geneva: World Health Organization; 2001.

12. Lima CT, Freire ACC, Silva APB, Teixeira RM, Farrel M, Prince M. Concurrent and construct validity of the AUDIT in an urban Brazilian sample. Alcohol Alcohol. 2005;40(6):584-9.

13. Fiellin DA, Carrington RM, $O^{\prime}$ Connor PG. Screening for alcohol problems in primary care: a systematic review. Arch Intern Med. 2000;160(13):1977-89.

14. Amato TC, Silveira OS, Oliveira JS, Ronzani TM. Uso de bebida alcoólica, religião e outras características sociodemográficas em pacientes da Atenção Primária à Saúde - Juiz de Fora, MG, Brasil - 2006. SMAD, Rev Eletrônica Saude Mental Alcool Drog (Ed. Port.) [periódico na Internet] 2008 jul-dez [acessado 2012 jul 7]; 4:[cerca de 17 p.]. Disponível em: <http://www2.eerp.udp.br/resmad/artigos.asp>.

15. Mendoza AZ. 0 uso de álcool na adolescência, uma expressão de masculinidade [tese]. Ribeirão Preto: Escola de Enfermagem de Ribeirão Preto, Universidade de São Paulo; 2004.
16. Oliva AL. Apoio social para dependentes do álcool [dissertação]. Ribeirão Preto: Faculdade de Medicina de Ribeirão Preto, Universidade de São Paulo; 2007.

17. Matute RC, Pillon SC. Alcohol consumption by nursing students in Honduras. Rev Lat Am Enfermagem. 2008;16 Spec No:584-9.

18. Brasil. Vigitel Brasil 2009: Vigilância de fatores de risco e proteção para doenças crônicas por inquérito telefônico. Brasília: Ministério da Saúde; 2010.

19. Flóripes TMF. Beber se embriagando (binge drinking): estudo de uma população de estudantes universitários que fazem uso de álcool de risco [dissertação]. Botucatu: Faculdade de Medicina de Botucatu, Universidade Estadual Paulista; 2008.

20. Laranjeira R, Pinsky I, Zaleski M, Caetano R (Org.). I Levantamento Nacional sobre os Padrões de Consumo de Álcool na População Brasileira. Brasília: SENAD; 2007.

21. Serdula MK, Brewer RD, Gillespie C, Denny CH, Mokdak A. Trends in alcohol use and binge drinking, 1985-1999: results of a multi-state survey. Am J Prev Med. 2004;26(4):294-8.

22. Primo NLNP, Stein AT. Prevalência do abuso e da dependência de álcool em Rio Grande (RS): um estudo de base populacional. Rev Psiquiatr Rio Gd Sul. 2004;26(3):280-6.

23. Costa JSD, Silveira MF, Gazalle FK, Oliveira SS, Hallal PC, Menezes AMB, et al. Heavy alcohol consumption and associated factors: a population-based study. Rev Saúde Pública. 2004;38(2):284-91.

24. Karlamangla A, Zhou K, Reuben D, Greendale G, Moore A. Longitudinal trajectories of heavy drinking in adults in the United States of America. Addiction. 2006;101(1):91-9.

25. Blue I. Individual and contextual effects on mental health status in São Paulo, Brazil. Rev Bras Psiquiatr. 2000;22(3):116-23.

26. Barros MBA, Botega NJ, Dalgalarrondo P, Marín-León L, Oliveira HB. Prevalence of alcohol abuse and factors associated factors in a population-based study. Rev Saúde Pública. 2007:41(4):502-9.

27. Bertoluzzi MC, Traebert J, Loguercio A, Kehrig RT. Prevalência e perfil dos usuários de álcool de população adulta em cidade do Sul do Brasil. Ciênc Saúde Colet. 2010;15(3):679-85.

28. Vargas D, Oliveira MAF, Araújo EC. Prevalência de dependência alcoólica em serviços de atenção primária à saúde de Bebedouro, São Paulo, Brasil. Cad Saude Publica. 2009;25(8):1711-20.

29. Secretaria de Vigilância à Saúde. Política Nacional de Promoção da Saúde. Brasília: Ministério da Saúde; 2006.

30. Jomar RT, Paixão LAR, Abreu AMM. Alcohol Use Disorders Identification Test (AUDIT) e sua aplicabilidade na atenção primária à saúde. Rev APS. 2012;15(1):113-7. 